\title{
Elasto-Plastic Springback of Beams Subjected to Repeated Bending/Unbending Histories
}

\author{
Franc Kosel, Tomaz Videnic, Tadej Kosel, and Mihael Brojan
}

\author{
(Submitted November 6, 2009; in revised form June 15, 2010)
}

\begin{abstract}
This contribution investigated repeated elastoplastic pure plane bending/unbending process of beams made of material with an elastic-linear hardening rheological model. The attention is focused on beams with cross sections which have at least one axis of symmetry and are initially straight or have constant radius of curvature. Elastoplastic deflection states of beams after repeated bending/unbending process are determined using the large displacement theory. Experiments were conducted to verify the theory for beams made of aluminium alloy AA $5050-\mathrm{H3} 8$ with rectangular cross sections. It is shown that maximal relative difference between experimental and theoretical results in the case of a largely curved beams after repeated bending/unbending process is $1.27 \%$.
\end{abstract}

Keywords elastic-linear strain hardening model, elastoplastic deformations, repeated bending/unbending process, springback

\section{Introduction}

In metal forming, there is always a certain amount of springback deformation when the forming tools are removed. The desired shape of the object is, therefore, not obtained if the tools are created according to exact geometry of the formed part. One can expect difficulties when reducing the springback effect more than what is the normal practice today, since all available techniques are currently used and the springback still poses considerable problem in many forming applications, even with low strength materials. The common way to deal with the problem is to add special techniques to reduce the effect of springback, such as extra features in radii, using smaller radii, or varying blankholder force in the forming process (Ref 1). These techniques only reduce the effect of springback, but the formed part will always spring back by a certain amount. The only way to have the part to be formed to an exactly desired shape is to have a tool geometry which differs from the geometry of the desired final shape (Ref 2).

Traditionally, attempts to express springback can be found in handbook tables or in springback graphics. Even though such an approach is not applicable to complex geometries or to materials without a large database of experiments, new springback graphics are available in the literature (Ref 3). However, the less time-consuming and inexpensive method is an analytical approach. Many researchers have worked on models for springback prediction, and a few of them will be briefly discussed here. For example, Gardiner (Ref 4) derived a

Franc Kosel, Tomaz Videnic, Tadej Kosel, and Mihael Brojan, Laboratory for Non-Linear Mechanics, Faculty of Mechanical Engineering, University of Ljubljana, Aškerčeva 6, 1000 Ljubljana, Slovenia. Contact e-mail: tomaz.videnic@fs.uni-lj.si. simplified model for the springback of elastic, ideal-plastic metals under pure bending. Johnson and Yu (Ref 5) developed formulas for springback of beams and plates undergoing linear work hardening. Nowadays, many engineering problems, and springback is not an exception, can be solved using finite element methods, and a number of authors can be quoted in this respect (Ref 2, 6-11). If analytical closed form solutions are in agreement with experiments, then they are always preferred due to their simplicity (Ref 12-18). Generally speaking, analytical solutions are applied to relatively simple problems, while numerical techniques are used in more complex cases. While analytical solutions provide better insight and understanding of the involved physical phenomena, and enable a clear representation of the influence of the problem parameters, numerical solutions provide accuracy and the capability to deal with complex problems. An interesting approach was adopted by Kazan et al. (Ref 19) who developed a prediction model of springback using artificial neural network. Finite element methods were used to generate training data for neural network. Besides single bending/unbending processes, complex bending histories, like double-bend technique, are also frequently encountered in die-forming processes ( $\operatorname{Ref} 7,20-23)$ and are generally more difficult for theoretical study.

This contribution analyzes repeated elastoplastic plane, pure bending/unbending process of beams made of material with an elastic-linear hardening rheological model. Beams are initially straight or have constant radius of curvature and are repeatedly subjected to a constant bending moment $M_{\mathrm{pl}}$ and then unloaded. In the case of the curved beam, it is assumed that the initial radius of curvature $R_{Z}$ was created by a previous elastoplastic bending/unbending process. The attention is confined to beams that have cross sections with at least one axis of symmetry. Elastoplastic deflection states of beams after repeated bending/ unbending processes are determined using the large displacement theory. Experiments to verify the analytical model were conducted for beams made of aluminium alloy AA 5050-H38 with constant rectangular cross sections. Another interesting case which was studied is the straightening of curved beams. In this case, the question emerges at which radius the curved beam has to be bent in the opposite direction to achieve flattening in the longitudinal axis after the unloading. 


\section{Formulation of the Problem}

In modeling plastic behavior a useful idealization is to consider the material as linearly elastic up to the yield point $\sigma_{0}$, as shown in Fig. 1, and then exhibiting linear strain hardening at strains beyond yield point. Strains up to yield are recoverable, and in unloading the material follows along the same elastic line it followed during loading - this is conventional elastic response. But if the material is strained beyond the yield point, stress $\sigma_{1}$ in Fig. 1, the elastic-plastic straining takes place. When the unloaded elastic part of the straining $\varepsilon_{\mathrm{el}}$ vanishes, this is called the springback effect, and the plastic strain $\varepsilon_{\mathrm{pl}}$ remains as a residual strain. In the case of uniaxial loading and unloading the stress state is equal to zero, whereas in the case of bending residual stresses are found, i.e. internal stresses that remain even after the external loads have been removed. If the material is loaded again, the response is elastic up to the state at which the unloading began, which is $\sigma_{1}$. This means that during the loading-unloading process the material becomes harder, $\sigma_{1}>\sigma_{0}$, and the process is called strain hardening.

The mathematical model is based on the following assumptions:

- the cross section has at least one axis of symmetry;

- the vector of inner bending moment is constant along the longitudinal $x$-axis and acts in the direction of $y$-axis.

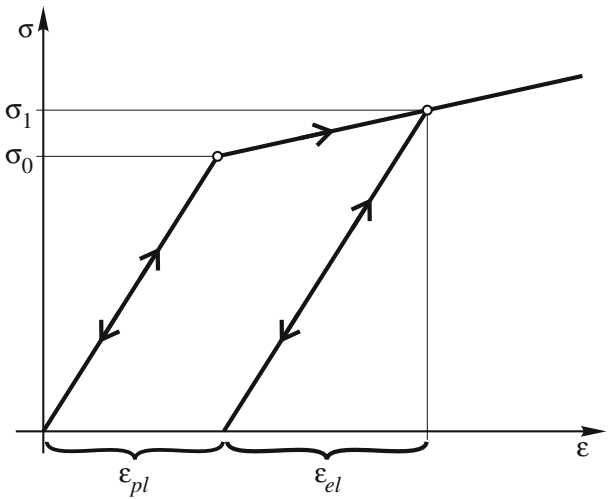

Fig. 1 Elastoplastic loading and unloading response
The $z$-axis is the symmetry axis of the cross section, see Fig. 2;

- the repeated loading process follow the elastic line of the stress-strain function up to the state at which the previous unloading began, stress $\sigma_{1}$ in Fig. 1;

- the beam is straight or has constant curvature before bending.

- the initial curvature of the curved beam was made by previous bending/unbending process of the straight beam;

- material of the beam is isotropic and homogeneous, and exhibits elastic-linear strain hardening rheological response;

- Bernoulli hypothesis of plane cross sections is used;

In elastoplasticity, it is well known that when the vertical $z$-axis of the cross section of the beam is a symmetry axis and $y$-axis in which the bending moment acts is not a symmetry axis, the neutral and centroidal axes do not coincide, Fig. 2. The distance between axes is denoted by $z_{\mathrm{s}}$. The neutral axis, in the case of elastoplastic stress state, is the axis where the bending stress is equal to zero, which in Fig. 2 is denoted by (N.axis)pl.

The position of neutral axis (N.axis)pl can be determined from the condition which states that the inner axial force $N$ caused by the bending moment $M_{\mathrm{pl}}$ is equal to zero. Owing to the action of the bending moment $M_{\mathrm{pl}}$ an elastoplastic stress state $\sigma_{\mathrm{pl}}=\sigma_{\mathrm{pl}}(z)$ occurs in the cross section of the beam, and the condition for determination of (N.axis)pl can be expressed as follows:

$N=\int_{A} \sigma_{\mathrm{pl}}(z) d \mathrm{~A}(z)=0$.

We used a special bending tool, Fig. 3, which enabled the application of constant bending moment $M_{\mathrm{pl}}$ along the beam's longitudinal axis ( $x$-axis). The tool is assembled from a circular plate with a radius $r_{0}$, on which a curved or straight beam is mounted, and a handle with a small wheel by which the beam can be curved along the circular plate (Ref 24).

After the bending process, the handle with a small wheel is removed and the deformed beam is released. Owing to the springback effect during unbending, the beam straightens to a radius of curvature $R$, see Fig. 3. This means that when the loading moment $M_{\mathrm{pl}}$ is removed, the elastic unloading moment $M_{\mathrm{el}}$ occurs, such that the resulting bending moment

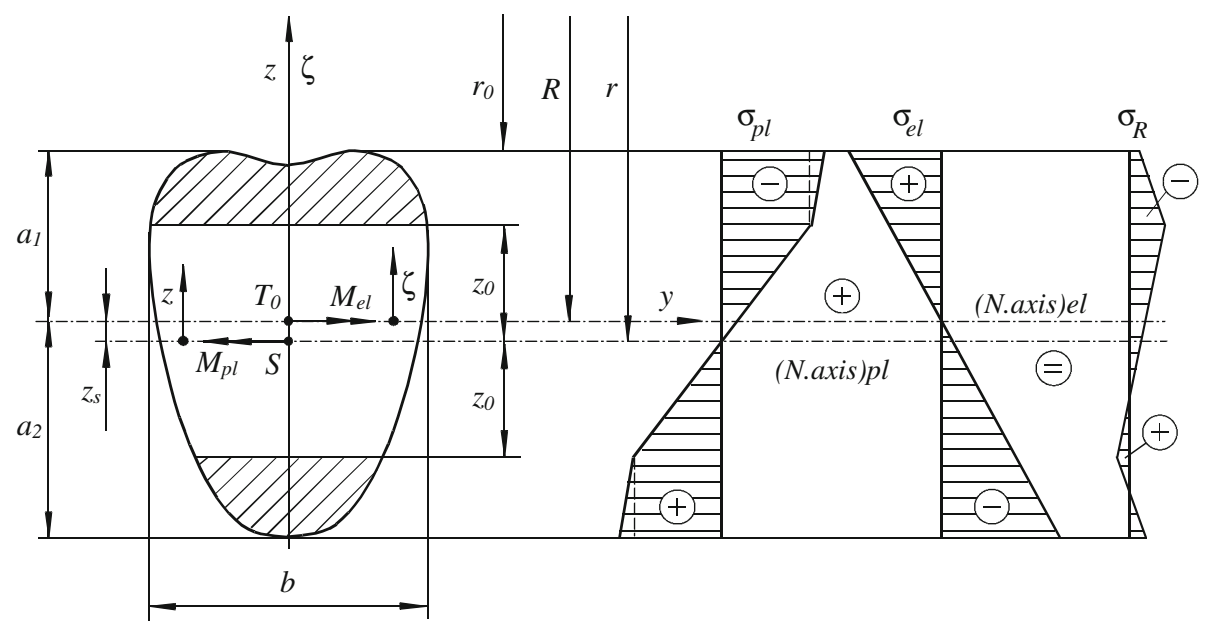

Fig. 2 Bending stress state in the cross section of the beam 


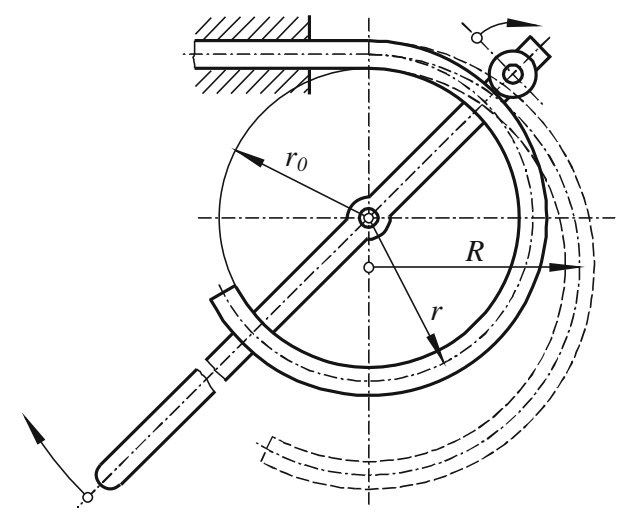

Fig. 3 Scheme of the special bending tool

$M_{\mathrm{R}}=M_{\mathrm{pl}}-M_{\mathrm{el}}=0$, i.e., the equilibrium in an unloaded state is restored. At this point, residual stress state $\sigma_{\mathrm{R}}$ is found, see Fig. 2

$M_{\mathrm{R}}=\int_{A} \sigma_{\mathrm{R}}(z) z d A(z)=0$.

During the unbending process, stress is decreasing linearly with respect to strains. Owing to the elastic bending moment $M_{\mathrm{el}}$, the elastic stress state $\sigma_{\mathrm{el}}$ occurs too. The neutral axis of the stress state $\sigma_{\text {el }}$, denoted by (N.axis)el in Fig. 2, coincides with the centroidal axis, which intersects $T_{0}$. It should also be noted that $\sigma_{\mathrm{el}}=\sigma_{\mathrm{el}}(\zeta)=M_{\mathrm{el}} \zeta / I_{y}$. Because of the relationship $\zeta=z-z_{\mathrm{s}}$, see Fig. 2, the elastic stress state can be expressed with coordinate $z$, as follows:

$\sigma_{\mathrm{el}}(z)=\frac{M_{\mathrm{el}}}{I_{y}}\left(z-z_{\mathrm{s}}\right)$,

where $I_{y}$ denotes a moment of inertia with respect to $y$-axis. The residual stress state $\sigma_{R}$ after the unloading process can be determined from

$$
\begin{aligned}
\sigma_{\mathrm{R}}(z) & =\sigma_{\mathrm{pl}}(z)-\sigma_{\mathrm{el}}(z) \\
& =\sigma_{\mathrm{pl}}(z)-\frac{M_{\mathrm{el}}}{I_{y}}\left(z-z_{\mathrm{s}}\right) .
\end{aligned}
$$

\section{Determination of the Radius of Curvature After Loading-Unloading Process}

In the case of pure bending, the inner moment is constant along the longitudinal axis of the beam, $M_{\mathrm{pl}}(x)=$ const., and consequently, the radius of curvature in the loaded state is also constant, $r(x)=r=$ const. For a curved beam with the initial curvature $1 / R_{Z}$, it is assumed that the beam was straight in the original state and that it was bent at least once in the elastoplastic domain to the value of the radius of the bending tool $r_{0}$. It can be seen from Fig. 2 that the relationship between the radius of curvature in the loaded state $r$ and radius of the bending tool $r_{0}$ is $r=r_{0}+a_{1}+z_{\mathrm{s}}$. After the elastic unbending, the curvature is reduced. The difference between the loaded and unloaded states is $1 / R_{\mathrm{el}}$, which can be expressed as

$\frac{1}{R_{\mathrm{el}}}=-\frac{\sigma_{\mathrm{el}}(\zeta)}{E \zeta}$

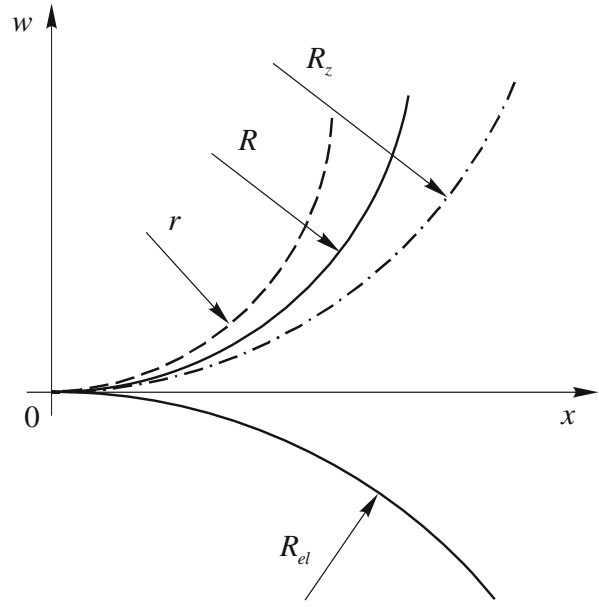

Fig. 4 Radii of curvatures in the loaded and unloaded states

The final displacement state of the beam after the bending/ unbending cycle is a curved beam with constant radius $R$. The difference in curvatures of the beam before and after the bending/unbending cycle is $1 / \rho$ and can be expressed according to the following equation, see also Fig. 4 ,

$\begin{aligned} \frac{1}{\rho} & =\frac{1}{R}-\frac{1}{R_{Z}} \\ & =\frac{1}{r}+\frac{1}{R_{\mathrm{el}}}-\frac{1}{R_{Z}} .\end{aligned}$

The distance from the neutral axis and boundary between elastic and plastic domain in the cross section of the beam is denoted by $z_{0}$, see Fig. 2, and can be calculated from

$\frac{1}{r}-\frac{1}{R_{Z}}=\frac{\sigma_{0}}{E z_{0}}$.

It is obvious that the beam undergoes partial plastification when $z_{0}<\max \left\{a_{1}, a_{2}\right\}$.

Combining Eq 5-7 results in

$\frac{1}{\rho}=\frac{\sigma_{0}}{E z_{0}}-\frac{\sigma_{\mathrm{el}}(\zeta)}{E \zeta}$,

from where the elastic bending stress $\sigma_{\mathrm{el}}(\zeta)$ during the process of unbending is obtained:

$\sigma_{\mathrm{el}}(\zeta)=\left(\frac{\sigma_{0}}{z_{0}}-\frac{E}{\rho}\right) \zeta$.

According to the concepts and assumptions mentioned in the previous sections, the stress state of the beam in the $i$-th ( $i \geq 1$ ) bending/unbending cycle can be determined by the following procedure.

Cycle 1: Initially straight beam with the radius of curvature $R_{Z}=R_{Z 1}=\infty$ is elasto-plastically bent with the moment $M_{\mathrm{pl}}$. The radius of curvature in the loaded state is $r=r_{1}=r_{01}+$ $a_{1}+z_{\mathrm{s} 1}$. The boundary between the elastic and plastic domains of the cross section is $z_{01}$ and can be calculated from Eq 7, i.e. $z_{0}=z_{01}=r_{1} \sigma_{0} / E$. After elastic unbending, the radius of curvature is $R=R_{1}$ and is taken as the initial radius of curvature for the second bending/unbending cycle: $R_{Z 2}=R_{1}$.

Cycle $i$ : The beam with the initial radius of curvature $R_{Z}=R_{Z i}=R_{i-1}$ is elasto-plastically bent with the moment $M_{\mathrm{pl}}$. 
The radius of curvature in the loaded state is $r=r_{i}=$ $r_{0 i}+a_{1}+z_{\mathrm{s} i}$ and the boundary between elastic and plastic domain $z_{0 i}$ can be calculated from

$\frac{1}{r_{i}}-\frac{1}{R_{i-1}}=\frac{\sigma_{0}}{E z_{0 i}}$.

After elastic unbending, the radius of curvature is $R_{i}$ and can be taken as the initial radius of the curvature for $i+1$ bending/ unbending cycle. The final curvature difference $1 / \rho_{i}$ after the elastic unbending process is:

$\frac{1}{\rho_{i}}=\frac{1}{R_{i}}-\frac{1}{R_{i-1}}$.

The elastic bending stress state $\sigma_{\mathrm{el}}(\zeta)$ during the elastic unbending process is

$\sigma_{\mathrm{el}}(\zeta)_{i}=\left(\frac{\sigma_{0}}{z_{0 i}}-\frac{E}{\rho_{i}}\right) \zeta$.

The elastoplastic stress state can be described, see Fig. 2:

$$
\begin{aligned}
& \sigma_{\mathrm{pl}}(z)_{i}=\sigma_{0}-E_{\mathrm{t}} k_{i}\left(z+z_{0 i}\right), \quad-\left(a_{2}-z_{\mathrm{si}}\right) \leq z \leq-z_{0 i} \\
& \sigma_{\mathrm{pl}}(z)_{i}=-E k_{i} z, \quad-z_{0 i} \leq z \leq z_{0 i} \\
& \sigma_{\mathrm{pl}}(z)_{i}=-\left(\sigma_{0}+E_{\mathrm{t}} k_{i}\left(z-z_{0 i}\right)\right), \quad z_{0 i} \leq z \leq\left(a_{1}+z_{\mathrm{si}}\right),
\end{aligned}
$$

where the curvature difference $k_{i}$ with regard to the neutral axis of the cross section with radius $r=r_{i}=r_{0 i}+a_{1}+z_{\mathrm{s} i}$ is

$k_{i}=\frac{1}{r_{i}}-\frac{1}{R_{i-1}}$.

The position of the neutral axis with regard to the centroid of the cross section in the $i$-th case of bending $z_{\mathrm{s} i}$ can be determined from Eq 1 and 13:

$$
\begin{aligned}
N= & -\int_{-z_{0 i}}^{+z_{0 i}} E k_{i} z d A(z) \\
& +\int_{-\left(a_{2}-z_{\mathrm{si}}\right)}^{\left(a_{1}+z_{\mathrm{si}}\right)}\left[\sigma_{0}-E_{\mathrm{t}} k_{i}\left(z+z_{0 i}\right)\right] d A(z) \\
& -\int_{z_{0 i}}^{-}\left[\sigma_{0}+E_{\mathrm{t}} k_{i}\left(z-z_{0 i}\right)\right] d A(z)=0 .
\end{aligned}
$$

The final radius of curvature in the $i$-th cycle of the bending/ unbending process can be determined from Eq 2 where the resulting stress $\sigma_{\mathrm{R}}(z)_{i}$ is

$\sigma_{\mathrm{R}}(z)_{i}=\sigma_{\mathrm{pl}}(z)_{i}+\sigma_{\mathrm{el}}(\zeta)_{i}$,

and the resulting bending moment after the bending/unbending process:

$$
\begin{aligned}
M_{\mathrm{R}}=M_{\mathrm{pl}}+M_{\mathrm{el}} & =\int_{A} \sigma_{\mathrm{R}}(z)_{i} z d A(z) \\
& =\int_{A} \sigma_{\mathrm{pl}}(z)_{i} z d A(z)+\int_{A} \sigma_{\mathrm{el}}(\zeta)_{i} \zeta d A(\zeta)=0 .
\end{aligned}
$$

Considering the expressions for elastic stress state, Eq 12, and elastoplastic stress state, Eq 13, the bending moment equilibrium equation after the bending/unbending process in the $i$-th cycle can be written:

$$
\begin{aligned}
M_{\mathrm{R}}= & \int_{-a_{2}}^{+a_{1}}\left[\frac{\sigma_{0}}{z_{0 i}}-\frac{E}{\rho_{i}}\right] \zeta^{2} d A(\zeta)+\int_{-\left(a_{2}-z_{\mathrm{si}}\right)}^{-z_{0 i}}\left[\sigma_{0}-E_{\mathrm{t}} k_{i}\left(z+z_{0 i}\right)\right] z d A(z) \\
& -\int_{-z_{0 i}}^{+z_{0 i}} E k_{i} z^{2} d A(z)-\int_{z_{0 i}}^{\left(a_{1}+z_{\mathrm{si}}\right)}\left[\sigma_{0}+E_{\mathrm{t}} k_{i}\left(z-z_{0 i}\right)\right] z d A(z)=0 .
\end{aligned}
$$

From Eq 18, the radius of curvature $R_{i}$ after the bending/ unbending process in the $i$-th cycle can be written in the following form:

$\frac{1}{R_{i}}=I_{i}+\frac{1}{R_{i-1}}$,

where

$$
\begin{aligned}
I_{i}= & \frac{1}{\int_{-a_{2}}^{+a_{1}} E \zeta^{2} d A(\zeta)}\left(\int_{-a_{2}}^{+a_{1}} \frac{\sigma_{0}}{z_{0 i}} \zeta^{2} d A(\zeta)-\int_{-z_{0 i}}^{+z_{0 i}} E k_{i} z^{2} d A(z)\right. \\
& +\int_{-\left(a_{2}-z_{s i}\right)}^{-z_{0 i}}\left[\sigma_{0}-E_{\mathrm{t}} k_{i}\left(z+z_{0 i}\right)\right] z d A(z) \\
& \left.-\int_{z_{0 i}}^{a_{1}+z_{s i}}\left[\sigma_{0}+E_{\mathrm{t}} k_{i}\left(z-z_{0 i}\right)\right] z d A(z)\right) .
\end{aligned}
$$

It is very interesting to determine the straightening radius $r_{r}$ to which the beam with initial radius $R_{Z}$ has to be elastoplastically bent in the opposite direction, so that the beam straightens after the elastic unbending process. In this case, the final radius after the unloading process is $R=\infty$, and from Eq 19 follows:

$\frac{1}{R}=I_{r}+\frac{1}{R_{Z}}=0$

where

$$
\begin{aligned}
I_{r}= & \frac{1}{\int_{-a_{2}}^{+a_{1}} E \zeta^{2} d A(\zeta)}\left(\int_{-a_{2}}^{+a_{1}} \frac{\sigma_{0}}{z_{0}} \zeta^{2} d A(\zeta)-\int_{-z_{0}}^{+z_{0}} E k_{r} z^{2} d A(z)\right. \\
& +\int_{-\left(a_{2}-z_{\mathrm{s}}\right)}^{-z_{0}}\left[\sigma_{0}-E_{\mathrm{t}} k_{r}\left(z+z_{0}\right)\right] z d A(z) \\
& \left.-\int_{z_{0}}^{a_{1}+z_{\mathrm{s}}}\left[\sigma_{0}+E_{\mathrm{t}} k_{r}\left(z-z_{0}\right)\right] z d A(z)\right) .
\end{aligned}
$$

The straightening curvature $k_{r}$ is defined as $k_{r}=1 / r_{r}-1 / R_{Z}$ and can be determined from Eq 21 and 22. Finally, the radius of the bending tool $r_{0 r}$ needed to straighten the beam with initial curvature $1 / R_{Z}$ can be calculated from

$r_{0 r}=\left(k_{r}+\frac{1}{R_{Z}}\right)^{-1}-a_{1}-z_{\mathrm{s}}$. 


\section{Some Cases of Elastoplastic Bending Process}

Using the above procedure for the determination of the elements of the elastoplastic bending/unbending process, the cross sections with rectangular, circular and isosceles-trapezoid shapes were selected, Fig. 5.

\subsection{Rectangular Cross Section}

The position of the neutral axis with regard to the centroid of the cross section in the $i$-th case of bending $z_{\mathrm{s} i}$ can be determined from $\mathrm{Eq} 15$ :

$$
\begin{aligned}
N= & -\int_{-z_{0 i}}^{+z_{0 i}} E k_{i} z b d z+\int_{-\left(a-z_{\mathrm{si}}\right)}^{-z_{0 i}}\left[\sigma_{0}-E_{\mathrm{t}} k_{i}\left(z+z_{0 i}\right)\right] b d z \\
& -\int_{z_{0 i}}^{a+z_{\mathrm{si}}}\left[\sigma_{0}+E_{\mathrm{t}} k_{i}\left(z-z_{0 i}\right)\right] b d z=0,
\end{aligned}
$$

where $a=a_{1}=a_{2}$ and $d A=b d z$ is used. After integration, the following expression can be deduced:

$z_{\mathrm{si}}\left(\sigma_{0}+E_{\mathrm{t}} k_{i}\left(a-z_{0 i}\right)\right)=0$.

Since $\sigma_{0}+E_{\mathrm{t}} k_{i}\left(a-z_{0 i}\right)$ cannot be zero, the position of the neutral axis $z_{\mathrm{s} i}=0$ in the case of rectangular cross section. It can be seen from Fig. 2 that, in this case, $z=\zeta$ and $r_{i}=r_{0 i}+a$.

The bending plastic moment $M_{\mathrm{pl}}$ can be calculated from Eq 17 and 18:

$$
\begin{aligned}
M_{\mathrm{pl}}= & -2 \int_{0}^{z_{0 i}} E k_{i} z^{2} b d z-2 \int_{z_{0 i}}^{a}\left(\sigma_{0}+E_{\mathrm{t}} k_{i}\left(z-z_{0 i}\right)\right) z b d z \\
M_{\mathrm{pl}}= & -\frac{2}{3} E k_{i} b z_{0 i}^{3}-\sigma_{0} b\left(a^{2}-z_{0 i}^{2}\right) \\
& -\frac{1}{3} E_{\mathrm{t}} k_{i} b\left(2 a^{3}-3 a^{2} z_{0 i}+z_{0 i}^{3}\right)
\end{aligned}
$$

The elastic unbending moment $M_{\mathrm{el}}$ can also be calculated from Eq 17 and 18:

$$
\begin{aligned}
& M_{\mathrm{el}}=2 \int_{0}^{a}\left(\frac{\sigma_{0}}{z_{0 i}}-\frac{E}{\rho_{i}}\right) z^{2} b d z, \\
& M_{\mathrm{el}}=\frac{2}{3} a^{3} b\left(\frac{\sigma_{0}}{z_{0 i}}-E\left(\frac{1}{R_{i}}-\frac{1}{R_{i-1}}\right)\right),
\end{aligned}
$$

The radius of curvature $R_{i}$ after the loading-unloading process in the $i$-th cycle can now be deduced from condition $M_{\mathrm{pl}}+M_{\mathrm{el}}=0, \mathrm{Eq} \mathrm{17}$, or directly from Eq 19 and 20:

$\frac{1}{R_{i}}=\left(k_{i}-\frac{3 \sigma_{0}}{2 E a}+\frac{1}{2 k_{i}^{2}}\left(\frac{\sigma_{0}}{E a}\right)^{3}\right)\left(1-\frac{E_{\mathrm{t}}}{E}\right)+\frac{1}{R_{i-1}}$.

In both cases, Eq 10 and 14 should also be used. From Eq 28, one can see that the width of the rectangular cross section $b$ does not influence the final radius $R_{i}$.

In a similar way as above, the expression for the determination of the straightening curvature $k_{r}$ can be calculated from Eq 21 and 22:

$$
\left(k_{r}-\frac{3}{2}\left(\frac{\sigma_{0}}{E a}\right)+\frac{1}{2 k_{r}^{2}}\left(\frac{\sigma_{0}}{E a}\right)^{3}\right)\left(1-\frac{E_{\mathrm{t}}}{E}\right)+\frac{1}{R_{z}}=0,
$$

and then the straightening radius of the bending tool $r_{0 r}$ from Eq 23, where $z_{\mathrm{s}}=0$ and $a_{1}=a$ should be used.

\subsection{Circular Cross Section}

In a similar way as in the case of rectangular cross section, the final radius $R_{i}$ of the beam with circular cross section can be calculated:

$$
\begin{aligned}
\frac{1}{R_{i}}= & \left(1-\frac{E_{\mathrm{t}}}{E}\right)\left\{k_{i}\left[1-\frac{2}{\pi} \arcsin \left(\frac{\sigma_{0}}{E a k_{i}}\right)\right]\right. \\
& \left.-\frac{2}{3 \pi}\left(\frac{\sigma_{0}}{E a}\right) \sqrt{1-\left(\frac{\sigma_{0}}{E a k_{i}}\right)^{2}}\left[5-2\left(\frac{\sigma_{0}}{E a k_{i}}\right)^{2}\right]\right\}+\frac{1}{R_{i-1}},
\end{aligned}
$$

where $a=a_{1}=a_{2}$ and $z_{\mathrm{s} i}=0$.

The expression for the determination of the straightening curvature $k_{r}$ is calculated from Eq 21 and 22:

$$
\begin{aligned}
& \left(1-\frac{E_{\mathrm{t}}}{E}\right)\left\{k_{r}\left[1-\frac{2}{\pi} \arcsin \left(\frac{\sigma_{0}}{E a k_{r}}\right)\right]\right. \\
& \left.-\frac{2}{3 \pi}\left(\frac{\sigma_{0}}{E a}\right) \sqrt{1-\left(\frac{\sigma_{0}}{E a k_{r}}\right)^{2}}\left[5-2\left(\frac{\sigma_{0}}{E a k_{r}}\right)^{2}\right]\right\}+\frac{1}{R_{z}}=0,
\end{aligned}
$$

and then the straightening radius of the bending tool $r_{0 r}$ from Eq 23, where $z_{\mathrm{s}}=0$ and $a_{1}=a$ should be used.

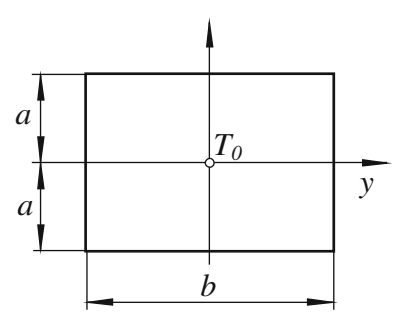

(a)

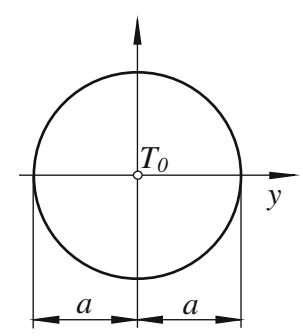

(b)

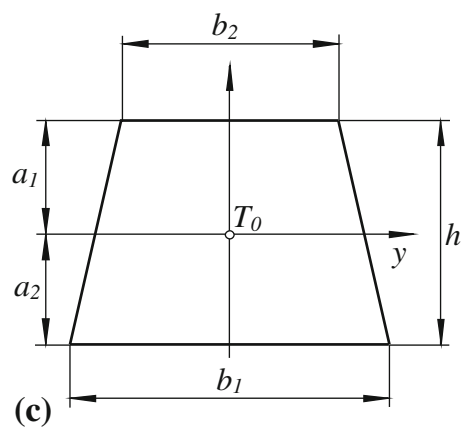

Fig. 5 Cross sections of beams 


\subsection{Isosceles Trapezoid Cross Section}

Isosceles trapezoid cross section is symmetrical only to $\zeta$ axis, whereas the $y$ axis, where the bending moment is acting, is asymmetrical. In this case, the neutral axis and centre of gravity of the cross section do not coincide; therefore it is $z_{\mathrm{s} i} \neq 0$ and $\zeta \neq z$, Fig. 2. The equation for determination of the position of the neutral axis with regard to the centroid of the cross section in the $i$-th case of bending $z_{\mathrm{s} i}$ can be determined from Eq 15:

$$
\begin{aligned}
z_{\mathrm{s} i}^{2} & \frac{\Delta b}{h}\left(1-\frac{E_{\mathrm{t}}}{E}\right)+z_{\mathrm{si} i}\left\{2\left(1-\frac{E_{\mathrm{t}}}{E}\right)\left(\frac{\Delta b}{h} a_{1}+b_{2}\right)\right. \\
+ & \left.\frac{E_{\mathrm{t}} k_{i}}{\sigma_{0}}\left[\left(a_{1}+a_{2}\right)\left(\frac{\Delta b}{h} a_{1}+b_{2}\right)+\frac{\Delta b}{h} \frac{a_{2}^{2}-a_{1}^{2}}{2}\right]\right\} \\
& -\left\{\left(1-\frac{E_{\mathrm{t}}}{E}\right)\left[\left(a_{2}-a_{1}\right)\left(\frac{\Delta b}{h} a_{1}+b_{2}\right)+\frac{\Delta b}{h} \frac{a_{1}^{2}+a_{2}^{2}}{2}\right]\right. \\
+ & \frac{E_{\mathrm{t}} k_{i}}{\sigma_{0}}\left[\frac{\Delta b}{3 h}\left(\frac{\sigma_{0}}{E k_{i}}\right)^{3}+\frac{a_{2}^{2}-a_{1}^{2}}{2}\left(\frac{\Delta b}{h} a_{1}+b_{2}\right)+\frac{\Delta b}{h} \frac{a_{1}^{3}+a_{2}^{3}}{3}\right] \\
- & \left.\frac{\Delta b}{3 h}\left(\frac{\sigma_{0}}{E k_{i}}\right)^{2}\right\}=0,
\end{aligned}
$$

where $\Delta b=b_{1}-b_{2}$ and $k_{i}=1 /\left(r_{0 i}+a_{1}+z_{\mathrm{s} i}\right)-1 / R_{i-1}$. The expression of the final radius $R_{i}$ follows from Eq 19 and 20: that $R_{i}=R_{i-1}$. In the case of isosceles trapezoid cross section, it follows from the Eq 32 that $z_{\mathrm{s} i}=0$, and the final radius after elastic unbending process is also equal to the initial one: $R_{i}=R_{i-1}$.

\section{Some Numerical Cases}

For the numerical and experimental cases of the elastoplastic bending, beams made of aluminium alloy AA 5050-H38 were chosen. The rheological properties of the material are expressed with stress-strain curves, determined by using a Zwick Z050 electronic measurement device equipped with Multisens extensometers, nominal force $50 \mathrm{kN}$, crosshead travel resolution $0.5 \mu \mathrm{m}$ and measurement range error $0.5 \%$ from $1 / 50$ of the nominal force. The measured stress-strain curves were then idealized into an elastic-linear strain hardening rheological model.

The data of the rectangular cross section are: $a=1.5 \mathrm{~mm}$, $b=20 \mathrm{~mm}$, the radius of the circular cross section is $a=2 \mathrm{~mm}$ and data for isosceles trapezoid cross section are: $b_{1}=5 \mathrm{~mm}$, $b_{2}=1.5 \mathrm{~mm}, h=3 \mathrm{~mm}$. The experimental stress-strain curves of five specimens made of aluminium alloy AA 5050-H38 are illustrated in Fig. 6. All the five tests were performed at the same experimental conditions, and the idealized data which are needed in the model are: Young modulus $E=67910 \mathrm{~N} / \mathrm{mm}^{2}$,

$$
\begin{aligned}
\frac{1}{R_{i}}= & \frac{12 k_{i}\left(1-\frac{E_{\mathrm{t}}}{E}\right)}{\frac{\Delta b}{h}\left[a_{1}^{4}+a_{2}^{3}\left(4 a_{1}+3 a_{2}\right)\right]+4 b_{2}\left(a_{1}^{3}+a_{2}^{3}\right)}\left\{\left[b_{2}+\frac{\Delta b}{h}\left(a_{1}+z_{\mathrm{si}}\right)\right]\left[\frac{1}{3}\left(\frac{\sigma_{0}}{E k_{i}}\right)^{3}-\frac{1}{2}\left(\frac{\sigma_{0}}{E k_{i}}\right)\left[\left(a_{1}+z_{\mathrm{si}}\right)^{2}+\left(a_{2}-z_{\mathrm{si}}\right)^{2}\right]\right]\right. \\
& \left.+\frac{1}{3}\left(\frac{\sigma_{0}}{E k_{i}}\right) \frac{\Delta b}{h}\left[\left(a_{1}+z_{\mathrm{s} i}\right)^{3}-\left(a_{2}-z_{\mathrm{s} i}\right)^{3}\right]\right\} \\
& +k_{i}\left\{1-\frac{E_{\mathrm{t}}}{E} \frac{\frac{\Delta b}{h}\left[\left(a_{1}+z_{\mathrm{si}}\right)^{4}+\left(a_{2}-z_{\mathrm{si}}\right)^{3}\left(4 a_{1}+3 a_{2}+z_{\mathrm{s} i}\right)\right]+4 b_{2}\left[\left(a_{1}+z_{\mathrm{si}}\right)^{3}+\left(a_{2}-z_{\mathrm{si}}\right)^{3}\right]}{\frac{\Delta b}{h}\left[a_{1}^{4}+a_{2}^{3}\left(4 a_{1}+3 a_{2}\right)\right]+4 b_{2}\left[a_{1}^{3}+a_{2}^{3}\right]}\right\}+\frac{1}{R_{i-1}} .
\end{aligned}
$$

The expression for the determination of the straightening curvature $k_{r}$ can be calculated from Eq 21 and 22: tangent modulus in the plastic domain $E_{\mathrm{t}}=550 \mathrm{~N} / \mathrm{mm}^{2}$, and yield stress $\sigma_{0}=199.77 \mathrm{~N} / \mathrm{mm}^{2}$.

$$
\begin{aligned}
& \frac{12 k_{r}\left(1-\frac{E_{\mathrm{t}}}{E}\right)}{\frac{\overline{\Delta b}}{h}\left[a_{1}^{4}+a_{2}^{3}\left(4 a_{1}+3 a_{2}\right)\right]+4 b_{2}\left(a_{1}^{3}+a_{2}^{3}\right)}\left\{\left[b_{2}+\frac{\Delta b}{h}\left(a_{1}+z_{\mathrm{si}}\right)\right]\left[\frac{1}{3}\left(\frac{\sigma_{0}}{E k_{r}}\right)^{3}-\frac{1}{2}\left(\frac{\sigma_{0}}{E k_{r}}\right)\left[\left(a_{1}+z_{\mathrm{si}}\right)^{2}+\left(a_{2}-z_{\mathrm{si}}\right)^{2}\right]\right]\right. \\
& \left.+\frac{1}{3}\left(\frac{\sigma_{0}}{E k_{r}}\right) \frac{\Delta b}{h}\left[\left(a_{1}+z_{\mathrm{si}}\right)^{3}-\left(a_{2}-z_{\mathrm{si}}\right)^{3}\right]\right\} \\
& +k_{r}\left\{1-\frac{E_{\mathrm{t}}}{E} \frac{\frac{\Delta b}{h}\left[\left(a_{1}+z_{\mathrm{si}}\right)^{4}+\left(a_{2}-z_{\mathrm{si}}\right)^{3}\left(4 a_{1}+3 a_{2}+z_{\mathrm{si}}\right)\right]+4 b_{2}\left[\left(a_{1}+z_{\mathrm{si}}\right)^{3}+\left(a_{2}-z_{\mathrm{si}}\right)^{3}\right]}{\frac{\Delta b}{h}\left[a_{1}^{4}+a_{2}^{3}\left(4 a_{1}+3 a_{2}\right)\right]+4 b_{2}\left[a_{1}^{3}+a_{2}^{3}\right]}\right\}+\frac{1}{R_{z}}=0,
\end{aligned}
$$

and then the straightening radius of the bending tool $r_{0 r}$ from Eq 23.

If, in Eq 32-34, the parameter $b_{2}$ is chosen as zero, then the expressions for $z_{\mathrm{s} i}, R_{i}$ and $k_{r}$ in the case of isosceles-triangular cross section can be written.

One can see that the structures of Eq 28, 30, and 33 for the determination of the final radii $R_{\mathrm{i}}$ after $i$-th elastoplastic bending/unbending cycle have the form which enables a simple analysis of the bending process.

In the case of elastic bending, it holds that $E_{\mathrm{t}}=E$. For the rectangular and circular cross sections, it follows right away
The numerical results of the bending process of the beams with rectangular, circular, and isosceles trapezoid cross sections are presented in Table 1-3, respectively.

The tables show the final radii after unloading process $R_{i}$, the boundary between elastic and plastic domain $z_{0 i}$, and for the isosceles trapezoid cross section, the neutral axis position $z_{\mathrm{s} i}$. The results of bending of beams with a straight initial longitudinal axis: $i=1, R_{Z 1}=\infty$, and the results of repeated bending process $i=1,2,3$ are presented. The beams were subjected to repeated bendings with tool radii $r_{0 i}=110,60$, and $37.5 \mathrm{~mm}$. 


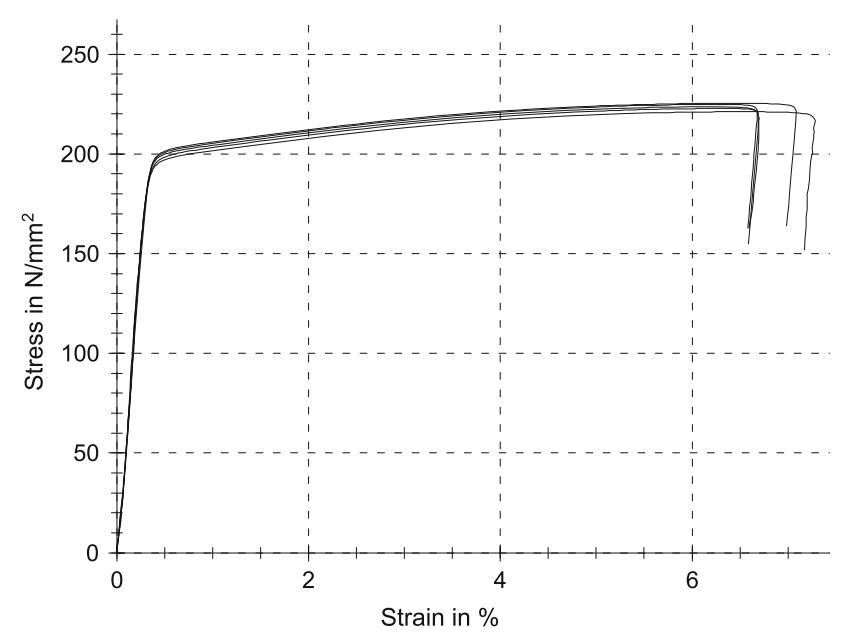

Fig. 6 Stress-strain curves of aluminium alloy AA 5050-H38

Table 1 Bending process of beam with rectangular cross section $3 \times 20 \mathrm{~mm}$

\begin{tabular}{|c|c|c|c|c|}
\hline$R_{Z i}, \mathbf{m m}$ & $r_{0 i}, \mathrm{~mm}$ & $z_{0 i}, \mathrm{~mm}$ & $i$ & $R_{i}, \mathbf{m m}$ \\
\hline \multirow[t]{3}{*}{$\infty$} & 110 & 0.3280 & 1 & 165.9857 \\
\hline & 60 & 0.1809 & 1 & 75.6158 \\
\hline & 37.5 & 0.1147 & 1 & 44.4026 \\
\hline$\infty$ & 110 & 0.3280 & 1 & 165.9857 \\
\hline 165.9857 & 60 & 0.2874 & 2 & 75.2156 \\
\hline \multirow[t]{2}{*}{75.2156} & 37.5 & 0.2383 & 3 & 44.1546 \\
\hline & & \multicolumn{2}{|c|}{$R_{Z}, \mathrm{~mm}$} & $r_{0 r}, \mathbf{m m}$ \\
\hline \multicolumn{2}{|l|}{$\begin{array}{l}\text { The radius of } \\
\text { bending tool } \\
\text { for beam } \\
\text { straightening }\end{array}$} & \multicolumn{2}{|c|}{$\begin{array}{r}165.9857 \\
75.6158 \\
44.4026\end{array}$} & $\begin{array}{l}386.7756 \\
355.7691 \\
362.3806\end{array}$ \\
\hline
\end{tabular}

The straightening radius of the bending tool $r_{0 r}$ for the beams with initial radius of curvature $R_{Z}$ was also calculated, which can be seen from Table 1-3. It should be noted here that, in this case, the straightening bending should be applied in the direction opposite to that of the initial curvature.

From the results, it can be concluded that the thickness of the beams has the crucial effect on the final radii after unloading $R_{i}$, while the width is not so important. The thicknesses of rectangular and isosceles trapezoid cross sections were taken as equal $(3 \mathrm{~mm})$ and the calculated values of the final radii after unloading $R_{i}$ and the straightening radius of the bending tool $r_{0 r}$ are quite similar even though the cross sections are very different. The diameter of the circular cross section was taken as $4 \mathrm{~mm}$, and it can be noted that especially the straightening radius $r_{0 r}$ differ significantly. It is interesting to find out that the boundary between elastic and plastic domain $z_{0 i}$ is similar in all the three cases.

\section{Experiments and Comparison of Experimental and Theoretical Results}

For experimental evaluation of the mathematical model, a special testing tool was constructed, Fig. 3 and 7, which
Table 2 Bending process of beam with circular cross section with diameter $4 \mathrm{~mm}$

\begin{tabular}{|c|c|c|c|c|}
\hline$R_{Z i}, \mathbf{m m}$ & $r_{0 i}, \mathrm{~mm}$ & $z_{0 i}, \mathrm{~mm}$ & $i$ & $R_{i}, \mathbf{m m}$ \\
\hline \multirow[t]{3}{*}{$\infty$} & 110 & 0.3295 & 1 & 155.9340 \\
\hline & 60 & 0.1824 & 1 & 73.8993 \\
\hline & 37.5 & 0.1162 & 1 & 44.1719 \\
\hline$\infty$ & 110 & 0.3295 & 1 & 155.9340 \\
\hline 155.9340 & 60 & 0.3028 & 2 & 73.5193 \\
\hline \multirow[t]{2}{*}{73.5193} & 37.5 & 0.2511 & 3 & 43.9284 \\
\hline & & \multicolumn{2}{|c|}{$\boldsymbol{R}_{Z}, \mathbf{m m}$} & $r_{0 r}, \mathbf{m m}$ \\
\hline \multirow{3}{*}{\multicolumn{2}{|c|}{$\begin{array}{l}\text { The radius of } \\
\text { bending tool } \\
\text { for beam } \\
\text { straightening } R=\infty\end{array}$}} & \multirow{3}{*}{\multicolumn{2}{|c|}{$\begin{array}{r}155.9340 \\
73.8993 \\
44.1719\end{array}$}} & 434.8926 \\
\hline & & & & 420.8527 \\
\hline & & & & 431.7254 \\
\hline
\end{tabular}

Table 3 Bending process of beam with isosceles trapezoid cross section $b_{1}=5 \mathrm{~mm}, b_{2}=1.5 \mathrm{~mm}$, $h=3 \mathbf{m m}$

\begin{tabular}{|c|c|c|c|c|c|}
\hline$R_{Z i}, \mathbf{m m}$ & $r_{0 i}, \mathbf{m m}$ & $z_{\mathrm{si}}, \mathrm{mm}$ & $z_{0 i}, \mathrm{~mm}$ & $i$ & $R_{i}, \mathbf{m m}$ \\
\hline \multirow[t]{3}{*}{$\infty$} & 110 & 0.1000 & 0.3291 & 1 & 168.9770 \\
\hline & 60 & 0.1012 & 0.1820 & 1 & 76.6774 \\
\hline & 37.5 & 0.0990 & 0.1158 & 1 & 45.0583 \\
\hline$\infty$ & 110 & 0.1000 & 0.3291 & 1 & 168.9770 \\
\hline 168.9770 & 60 & 0.1008 & 0.2871 & 2 & 76.2361 \\
\hline \multirow[t]{2}{*}{76.2361} & 37.5 & 0.1014 & 0.2395 & 3 & 44.7957 \\
\hline & & $z_{\mathrm{s}}, \mathbf{m m}$ & \multicolumn{2}{|c|}{$R_{Z}, \mathbf{m m}$} & $r_{0 r}, \mathbf{m m}$ \\
\hline \multirow{3}{*}{\multicolumn{2}{|c|}{$\begin{array}{l}\text { The radius of } \\
\text { bending tool } \\
\text { for beam } \\
\text { straightening } R=\infty\end{array}$}} & 0.1000 & \multirow{3}{*}{\multicolumn{2}{|c|}{$\begin{array}{r}168.9770 \\
76.6774 \\
45.0583\end{array}$}} & 388.4148 \\
\hline & & 0.1012 & & & 345.6818 \\
\hline & & 0.0990 & & & 350.8978 \\
\hline
\end{tabular}

enables pure elastoplastic bending/unbending of the beams. The testing unit also enables changing of the circular plates for a repeated bending/unbending process with different bending radii $r_{0 i}$. The following values of $r_{0 i}$ were chosen: 110,60 , and $37.5 \mathrm{~mm}$. Figure 7(a) shows bending to radius $r_{01}=110 \mathrm{~mm}$ of the initially straight beam with rectangular cross section, and Fig. 7(b) shows the same beam after the unloading process with the final radius $R_{1}=165.7 \mathrm{~mm}$. The second step, $i=2$, of bending to tool radius $r_{02}=60 \mathrm{~mm}$ of the same beam can be seen in Fig. 7(c), and the unloaded state after the second step of bending with the final radius $R_{2}=75.3 \mathrm{~mm}$ in Fig. $7(\mathrm{~d})$. The third bending step, $i=3$, with bending to radius $r_{03}=37.5 \mathrm{~mm}$ of the same beam can be seen in the loaded state in Fig. 7(e) and in the unloaded state in Fig. 7(f).

Table 4 presents experimental results of the final radii $\left(R_{i}^{\text {exp }}\right)$ of three beams with rectangular cross sections, $a=1.5 \mathrm{~mm}$ and $b=20 \mathrm{~mm}$, after repeated $(i=1,2,3)$ bending/unbending process. In Table 5, the average experimental values $R_{i}^{\exp }$ are compared to theoretically determined values, from Table 1, of the final radii of the beam $R_{i}$.

The relative difference $\delta_{i}$ between experimentally and theoretically determined values of the final radius, which is calculated from $\delta_{i}=100\left(R_{i}-R_{i}^{\exp }\right) / R_{i}$, can be seen from the last column in Table 5 . 

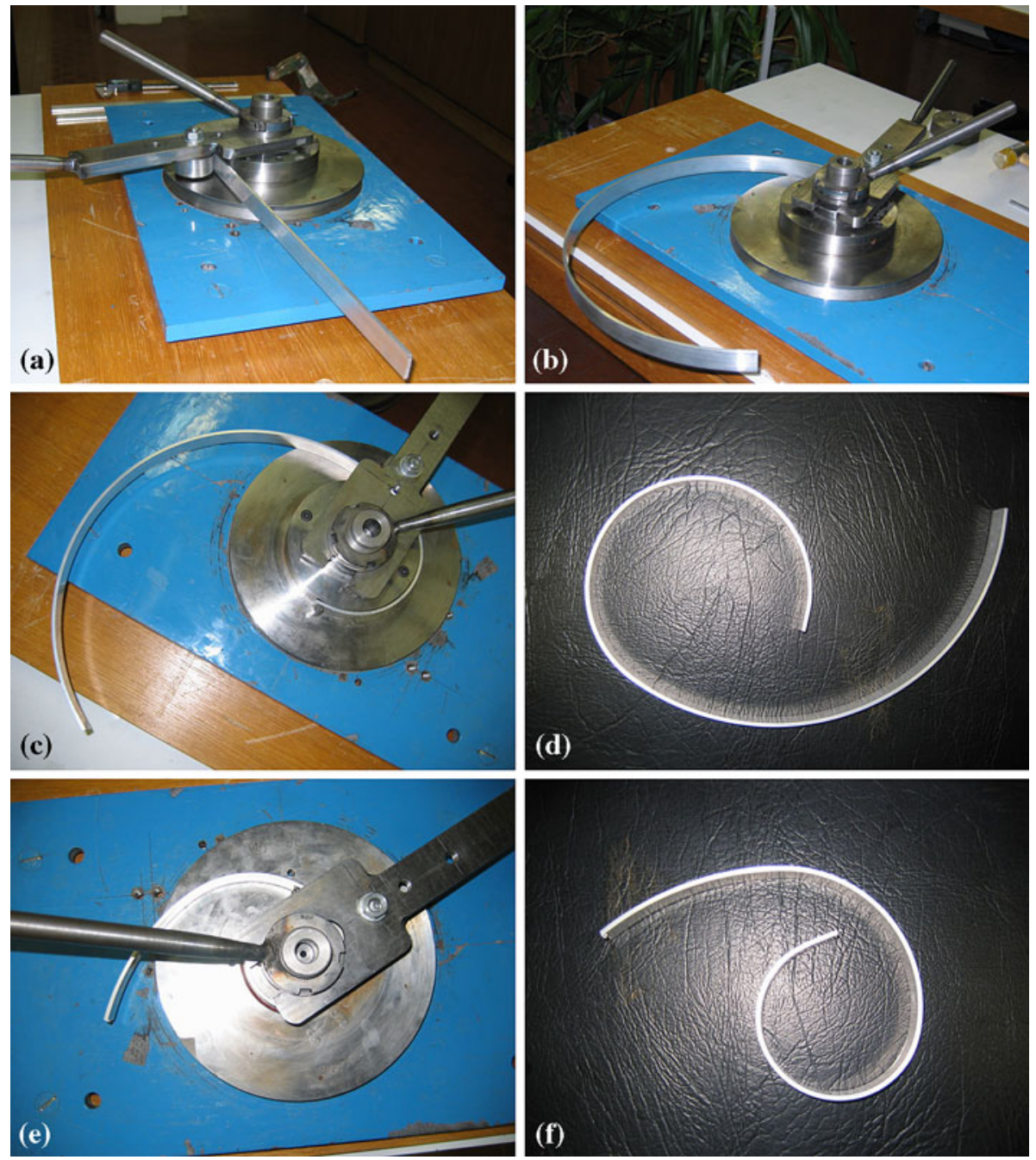

Fig. 7 (a) Bending of initially straight beam to radius $r_{01}=110 \mathrm{~mm}, i=1$. The initial radius $R_{Z 1}=\infty$. (b) The beam in unloaded state after the first cycle of bending, $i=1$. (c) The second repeated step of bending to radius $r_{02}=60 \mathrm{~mm}, i=2$. The initial radius $R_{Z 2}=R_{1}=165.7 \mathrm{~mm}$. (d) The beam in the unloaded state after the second cycle of bending, $i=2$. (e) The third repeated step of bending to radius $r_{03}=37.5 \mathrm{~mm}$, $i=3$. The initial radius $R_{Z 3}=R_{2}=75.3 \mathrm{~mm}$. (f) The beam in the unloaded state after the third cycle of bending, $i=3$

Table 4 The experimental results of repeated bending/ unbending of beams with rectangular cross section

\begin{tabular}{lllccc}
\hline $\boldsymbol{i}$ & $\boldsymbol{R}_{\boldsymbol{Z} \boldsymbol{i}}, \mathbf{m m}$ & $\boldsymbol{r}_{\mathbf{0} \boldsymbol{i}}, \mathbf{m m}$ & $\left(\boldsymbol{R}_{\boldsymbol{i}}^{\mathbf{e x p}}\right)_{\mathbf{1}}, \mathbf{m m}$ & $\left(\boldsymbol{R}_{\boldsymbol{i}}^{\mathbf{e x p}}\right)_{\mathbf{2}}, \mathbf{m m}$ & $\left(\boldsymbol{R}_{\boldsymbol{i}}^{\mathbf{e x p}}\right)_{\mathbf{3}}, \mathbf{m m}$ \\
\hline 1 & $\infty$ & 110 & 165.7 & 170.0 & 168.5 \\
2 & 168.1 & 60 & 75.3 & 74.8 & 74.5 \\
3 & 74.9 & 37.5 & 44.0 & 44.0 & 44.2 \\
\hline
\end{tabular}

Table 5 The comparison between average experimental values $R_{i}^{\text {exp }}$ and theoretically determined final radius $R_{i}$ after the first, the second, and the third bending/ unbending cycle

\begin{tabular}{rrrr}
\hline $\boldsymbol{i}$ & $\boldsymbol{R}_{\boldsymbol{i}}, \mathbf{m m}$ & $\boldsymbol{R}_{\boldsymbol{i}}^{\text {exp }}, \mathbf{m m}$ & $\delta_{\boldsymbol{i}}, \boldsymbol{\%}$ \\
\hline 1 & 165.9857 & 168.1 & -1.27 \\
2 & 75.2156 & 74.9 & +0.42 \\
3 & 44.1546 & 44.1 & +0.12 \\
\hline
\end{tabular}

\section{Conclusion}

Springback prediction is an important issue in metal forming industry. Most metal elements undergo a complicated cyclical deformation history during the forming process, and for an accurate prediction of springback, a simple and reliable analytical model can be useful.

In this article, we developed and experimentally verified such an analytical model to predict springback after repeated bending/unbending process. The procedure for determining the final radius of curvature of the beam is described. The condition that the form of cross section of the beam has to be symmetrical at least to $z$-axis ensures plane strain in $(x, z)$ plane. The expressions for determining the final radii of curvatures have the forms from which the influence of linear strain hardening effect in the plastic domain can be clearly seen.

From Table 1-3, it can be seen that, after the unloading process, the relative differences between final radii $R_{i}$ of beams that were initially straight and those that were bent repeatedly are very small. 
It is interesting to investigate the cases of straightening, when the repeatedly bent/unbent beam is then bent in the opposite direction in such a way that the beam straightens after unloading. In Table 1-3 are collected calculated values of radii of the bending tool $r_{0 r}$ needed to straighten the beam with the initial curvature $1 / R_{Z}$.

The comparison between the theoretically determined radii of curvature after unbending and the experimentally determined values of radii of curvature is shown by the relative difference $\delta_{i}$ in Table 5. In the case of repeated bending/unbending process of the initially straight beam with rectangular cross section, the largest relative difference between theoretically and experimentally determined final radius of curvature in the unloaded state is $1.27 \%$. It can be concluded that the analytical model presented is in good agreement with experiments.

\section{References}

1. G. Liu, Z. Lin, Y. Bao, and J. Cao, Eliminating Springback Error in U-Shaped Part Forming by Variable Blankholder Force, J. Mater. Eng. Perform., 2002, 11(1), p 64-70

2. A. Jernberg, A Method for Modifying the Forming Tool Geometry in Order to Compensate for Springback Effects, 4th European LS-DYNA Users Conference, May 22-23, 2003 (Ulm, Germany), DYNAmore 2003, p E-III-45-E-III-54

3. M.L. Garcia-Romeu, J. Ciurana, and I. Ferrer, Springback Determination of Sheet Metals in an Air Bending Process Based on an Experimental Work, J. Mater. Process. Technol., 2007, 191(1-3), p 174-177

4. F.J. Gardiner, The Springback of Metals, Trans. ASME, 1957, 79, p 1-9

5. W. Johnson and T.X. Yu, On Springback After the Pure Bending of Beams and Plates of Elastic Work-Hardening Materials III, Int. J. Mech. Sci., 1981, 23(11), p 687-695

6. K.P. Li, W.P. Carden, and R.H. Wagoner, Simulation of Springback, Int. J. Mech. Sci., 2002, 44(1), p 103-122

7. F. Yoshida and T. Uemori, A Model of Large-Strain Cyclic Plasticity and its Application to Springback Simulation, Int. J. Mech. Sci., 2003, 45(10), p 1687-1702

8. J. Wang, V. Levkovitch, F. Reusch, and B. Svendsen, On the Modelling and Simulation of Induced Anisotropy in Polycrystalline Metals with
Application to Springback, Arch. Appl. Mech., 2005, 74(11-12), p 890-899

9. M.G. Lee, D. Kim, C. Kim, M.L. Wenner, R.H. Wagoner, and K. Chung, A Practical Two-Surface Plasticity its Application to Spring-Back Prediction, Int. J. Plast., 2007, 23(7), p 1189-1212

10. D. Zeng and Z.C. Xia, A Modified Mroz Model for Springback Prediction, J. Mater. Eng. Perform., 2007, 16(3), p 293-300

11. F. Cimolin, R. Vadori, and C. Canuto, Springback Compensation in Deep Drawing Applications, Meccanica, 2008, 43(2), p 101-113

12. A.A. El-Domiaty and A.A. Elsharkawy, Stretch-Bending Analysis of U-Section Beams, Int. J. Mach. Tools Manuf., 1998, 38(1-2), p 75-95

13. H.A. Al-Qureshi, Elastic-Plastic Analysis of Tube Bending, Int. J. Mach. Tools Manuf., 1999, 39(1), p 87-104

14. K.C. Chan and S.H. Wang, Effect of Anisotropy on Springback of Integrated Circuit Leadframes, J. Mater. Eng. Perform., 1999, 8(3), p 368-374

15. S. Baragetti, A Theoretical Study on Nonlinear Bending of Wires, Meccanica, 2006, 41(4), p 443-458

16. Z. Dongjuan, C. Zhenshan, R. Xueyu, and L. Yuqiang, An Analytical Model for Predicting Springback and Side Wall Curl of Sheet after U-Bending, Comput. Mater. Sci., 2007, 38(4), p 707-715

17. A. El Megharbel, G.A. El Nasser, and A. El Domiaty, Bending of Tube and Section Made of Strain-Hardening Materials, J. Mater. Process. Technol., 2008, 203(1-3), p 372-380

18. M.G. Lee, J.H. Kim, K. Chung, S.J. Kim, R.H. Wagoner, and H.Y. Kim, Analytical Springback Model for Lightweight Hexagonal Close-Packed Sheet Metal, Int. J. Plast., 2009, 25(3), p 399-419

19. R. Kazan, M. Firat, and A.E. Tiryaki, Prediction of Springback in Wipe-Bending Process of Sheet Metal Using Neural Network, Mater. Des., 2009, 30(2), p 418-423

20. C.C. Chu, Elastic-Plastic Springback of Sheet Metals Subjected to Complex Plane Strain Bending Histories, Int. J. Solids Struct., 1986, 22(10), p 1071-1081

21. J.S. Shu and C. Hung, Finite Element Analysis and Optimization of Springback Reduction: The "Double-Bend" Technique, Int. J. Mach. Tools Manuf., 1996, 36(4), p 423-434

22. J.T. Gau and G.L. Kinzel, A New Model for Springback Prediction in which the Bauschinger Effect is Considered, Int. J. Mech. Sci., 2001, 43(8), p 1813-1832

23. Y. Yao, M.W. Lu, and X. Zhang, Elasto-Plastic Behavior of Pipe Subjected to Steady Axial Load and Cyclic Bending, Nucl. Eng. Des., 2004, 229(2-3), p 189-197

24. F. Kosel and I. Borštnik, Upogib nosilcev s konstantnim prerezom v elasto-plastičnem območju (Bending of Beams with the Constants Cross Sections in the Elastoplastic Domain), Strojniški vestnik (J. Mech. Eng.), 1979, 25(9-10), p 205-208 (in Slovene) 